\title{
Yield and Growth Parameters of Maize Hybrids As Influenced By Target Yield Approach
}

\author{
H.T. Chetan ${ }^{1 *}$, M.P. Potdar ${ }^{1}$, B.T. Nadagouda ${ }^{2}$ and Gurupada B. Balol ${ }^{3}$ \\ ${ }^{1}$ Department of Agronomy, UAS, Dharwad - 580 005, India \\ ${ }^{2}$ Department of Agronomy, ARS-Mudhol, UAS, Dharwad - 580 005, India \\ ${ }^{3}$ Department of Plant Pathology, AICRP on Chickpea, UAS, Dharwad - 580 005, India \\ *Corresponding author
}

\begin{tabular}{|c|c|}
\hline & A B S T R A C T \\
\hline $\begin{array}{l}\text { Hybrids, SSNM, } \\
\text { Target yield, } \\
\text { Phenolgical stages, } \\
\text { Growth parameters } \\
\text { and Yield. }\end{array}$ & \multirow{3}{*}{$\begin{array}{l}\text { Experiment was conducted during } 2014 \text { kharif season at the Agricultural Research Station, } \\
\text { Mudhol to investigate the response of two maize hybrids viz., NK-6240 and } 900 \mathrm{M} \text { GOLD } \\
\text { to five target yield levels }\left(100,120,140 \mathrm{q} \mathrm{ha}^{-1}, \mathrm{RDF} \text { and absolute control). The hybrid } 900\right. \\
\mathrm{M} \text { GOLD with target yield level of } 140 \mathrm{q} \mathrm{ha}^{-1} \text { recorded significantly higher growth } \\
\text { parameters namely plant height, leaf area, LAD, stem girth at different phenological } \\
\left.\text { stages. Significantly higher grain ( } 130.53 \mathrm{q} \mathrm{ha}^{-1}\right) \text { target yield level of } 140 \mathrm{q} \mathrm{ha}^{-1} \text {. The } \\
\text { increase in grain yield in hybrid } 900 \mathrm{M} \text { GOLD with target yield level of } 140 \mathrm{q} \mathrm{ha}^{-1} \text { could } \\
\text { be the maximum number of leaves per plant }(5.20,7.93,11.93,14.20 .11 .20,3.87 \text { at } 25 \text {, } \\
35,55,65 \text { and } 85 \mathrm{DAS} \text {, respectively), leaf area ( } 987.61,2167.8,5638,7308.14,4000.04 \\
\mathrm{~cm}^{2} \text { at } 25,35,55,65 \text { and } 85 \text { DAS, respectively), LAD }(13.15,65.05,53.94,94.23 \text { between } \\
25 \text { to } 35 \mathrm{DAS}, 35 \text { to } 55 \mathrm{DAS}, 55 \text { to } 65 \mathrm{DAS} \text { and } 65 \text { to } 85 \mathrm{DAS} \text {, respectively), stem girth } \\
\text { (3.25, } 4.50,8.68,8.75,9.10 \mathrm{~cm} \text { at } 25,35,55,65 \text { and } 85 \text { DAS, respectively) compared to } \\
\text { absolute control. }\end{array}$} \\
\hline Article Info & \\
\hline $\begin{array}{l}\text { Accepted: } \\
\text { 04 July } 2017 \\
\text { Available Online: } \\
\text { 10 September } 2017\end{array}$ & \\
\hline
\end{tabular}

\section{Introduction}

The productivity of any crop depends on its genetic potential, soil and climate. Since genotype is fixed character, growth and yield of a crop is influenced by environmental and cultural practices. Under specific agroclimatic condition, yield of a crop mainly depends on balanced nutrition.

Although plants get some amount of nutrients from soil, they are inadequate to meet the ever increasing demand for higher production. Improper nutrition leading to the nutrient imbalance in plants is a factor contributing to lower production.
The deficiencies of $\mathrm{N}, \mathrm{P}$ and $\mathrm{K}$ also pose a serious threat to our agricultural production.

Maize a thermo-insensitive, long day kharif cereal adopts well to the diverse climatic conditions. With the introduction of high yielding varieties of maize, use of high analysis chemical fertilizer and monocropping practices gained popularity. This led to systematic mining of soils for major nutrients such as nitrogen, phosphorus and potassium resulting in wide spread low harvest of poor quality grains. Adequate nutrition therefore, is important but the emphasis should also be 
given on the dosage in commensuration with the crop need pattern otherwise nutrients like nitrogen are subjected for loss resulting in low use efficiency of added nutrients besides low and poor quality produce.

The production of economic yield of crop is outcome of many complicated and yield related physiological, morphological and biochemical events taking place at different plant parts. These complicated events in turn, based on environment under which the crop is growing. The agronomic practice can modify the environment to certain extent and thereby help the crop to exploit the available resources efficiently to achieve higher production. Thus maximum yields are obtained when optimum conditions are provided for crop.

Yield being a complex character, it is influenced by many morphological characteristics and biochemical processes that occur during the crop growth and development.

Varying levels of nutrient management obviously results into greater variation in growth patterns of maize leading to different levels of yield. This is ascribed with higher growth and yield components which might be due to supply of all nutrients - major and minor elements by targeted yield approach (through inorganic fertilizer and INM) and their release was rather broad and could have met adequately the requirements of maize. Such a conducive effect of targeted yield approach could be attributed to the supply of nutrients through mineralization and improvement of physicochemical and biological properties of soil. Higher yield parameters can be attributed to the ability of targeted yield approaches to satisfy the nutrient demand of crop more efficiently than other inorganic manures as revealed in differences in nutrient uptake of maize.

\section{Materials and Methods}

A Field experiment was conducted during kharif 2014 at the Agricultural Research Station Mudhol, University of Agricultural Sciences, Dharwad. It is located in Bagalkot district in the northern part of Karnataka State at $16^{\circ} 20^{\prime \prime} \mathrm{N}$ latitude, $75^{\circ} 15^{\prime \prime} \mathrm{E}$ longitude and at an altitude of 577.6 meters above mean sea level. The soil of the experimental field was vertisol with $\mathrm{pH}$ 7.95. The treatment combinations comprising two maize hybrids viz., NK-6240 and 900 M GOLD as main plot treatments and five target yield levels viz., 100 (288.0:89.0:67.2 N, $\mathrm{P}_{2} \mathrm{O}_{5}$ and $\mathrm{K}_{2} \mathrm{O} \mathrm{kg}$ ha $\left.{ }^{1}\right), 120\left(345.6: 106.8: 80.6 \mathrm{~N}, \mathrm{P}_{2} \mathrm{O}_{5}\right.$ and $\mathrm{K}_{2} \mathrm{O} \mathrm{kg}$ ha $\left.^{-1}\right), 140$ q ha-1 (403.2:124.6:94.0 N, $\mathrm{P}_{2} \mathrm{O}_{5}$ and $\mathrm{K}_{2} \mathrm{O} \mathrm{kg} \mathrm{ha}{ }^{-1}$ ), recommended dose of fertilizer (RDF) (150.0:65.0:65.0 N, $\mathrm{P}_{2} \mathrm{O}_{5}$ and $\mathrm{K}_{2} \mathrm{O} \mathrm{kg} \mathrm{ha}{ }^{-1}$ ) and absolute control as sub plot treatments. Nutrients required (FA) to achieve target yield (T) was calculated by using the formulae.

FA $=$ Nutrient uptake by crop per quintal grain yield $\times \mathrm{T} \times$ per cent $\mathrm{EFR}$

$\mathrm{EFR}=20$ per cent more or less fertilizer to be applied as per the soil supply capacity for N, $\mathrm{P}_{2} \mathrm{O}_{5}$ and $\mathrm{K}_{2} \mathrm{O}$ as Low (20 per cent more than the calculated value), Medium (As per the calculated value) and High (20 percent less than the calculated value). Nutrient uptake by maize $\left(2.40 \mathrm{~kg} \mathrm{~N}, 0.89 \mathrm{~kg} \mathrm{P}_{2} \mathrm{O}_{5}\right.$ and $0.84 \mathrm{~kg}$ $\mathrm{K}_{2} \mathrm{O}$ ) to produce quintal of grains was worked out by referring previous works (Pagad, 2014) on maize at the same location and accordingly 288.0:89.0:67.2 N, $\mathrm{P}_{2} \mathrm{O}_{5}$ and $\mathrm{K}_{2} \mathrm{Okg} \mathrm{ha}{ }^{-1}$, 345.6:106.8:80.6 N, $\mathrm{P}_{2} \mathrm{O}_{5}$ and $\mathrm{K}_{2} \mathrm{O} \mathrm{kg}$ ha ${ }^{1}$,403.2:124.6:94.0 N, $\mathrm{P}_{2} \mathrm{O}_{5}$ and $\mathrm{K}_{2} \mathrm{O} \mathrm{kg} \mathrm{ha}{ }^{-1}$, was required to achieve target yield of 100 , 120 and $140 \mathrm{q}$ ha $^{-1}$ respectively. The experiment was laid out in split plot design with three replications. $10 \% \mathrm{~N}$ and entire dose of $\mathrm{P}_{2} \mathrm{O}_{5}$ and $\mathrm{K}_{2} \mathrm{O}$ was applied at the time of sowing in the form of urea, single super 
phosphate and muriate of potash respectively. The fertilizers were applied by placing along the lines $5 \mathrm{~cm}$ away and $5 \mathrm{~cm}$ below the seed rows. Sowing of maize was done on $6^{\text {th }}$ August 2014 with a spacing of $60 \mathrm{~cm}$ between rows and $20 \mathrm{~cm}$ between plants. The remaining amount of nitrogen was applied in four split dosage at $25(20 \% \mathrm{~N}), 35(30 \% \mathrm{~N})$, $55(30 \% \mathrm{~N})$ and at 65 DAS $(10 \% \mathrm{~N})$. All the plots were uniformly irrigated as and when required based on soil moisture content and phenological stages of the crop growth. Total three irrigations were provided to crop. Observation on growth parameters were collected at different phenological stages $\left[\mathrm{V}_{2}\right.$ Collar of $2^{\text {nd }}$ leaf visible, $V_{7}$ Collar of $7^{\text {th }}$ leaf visible, $\mathrm{V}_{12}$ Collar of $12^{\text {th }}$ leaf visible, $\mathrm{V}_{16}$ Collar of $16^{\text {th }}$ leaf visible (Tassel), Milky stage and at harvest]. Harvesting was done, when the sheath of the cob dried completely.

\section{Results and Discussion}

\section{Grain yield (q ha $\left.{ }^{-1}\right)$}

Grain yield of maize was significantly influenced by maize hybrids. The maize hybrid 900 M GOLD (93.47 q ha ${ }^{-1}$ ) recorded significantly higher grain yield over NK-6240 $\left(91.81 \mathrm{q} \mathrm{ha}^{-1}\right)$. The increase in grain yield was to the extent of 1.77 per cent over NK-6240 hybrid. This might be due to genetic makeup and morphological characteristics of hybrids in exploiting climatic optima at important growth stages (Jemal Abdulai, 2010).Similar kinds of result have also been reported by Daikho (2013) and Singh et al., (2014). Among different target levels, significantly higher grain yield was recorded with target yield level of $140 \mathrm{q} \mathrm{ha}^{-1}\left(86.34 \mathrm{q} \mathrm{ha}^{-1}\right)$ over target yield of $100,120 \mathrm{q} \mathrm{ha}^{-1}$, recommended dose of fertilizer (RDF) and absolute control. Significantly lower grain yield (19.01 q ha ${ }^{-1}$ ) was observed in absolute control. The increase in grain yield was 78.0 per cent over absolute control and 16.9, 7.7 and 39.8 per cent as compared to target yield levels of 100, $120 \mathrm{q} \mathrm{ha}^{-1}$ and RDF respectively. The higher grain maize was mainly due to better translocation of photosynthates from source to sink and higher growth attributing characters like higher number of leaves, leaf area and higher dry matter production and its accumulation into different parts of plant and yield attributing characters like cob length, cob girth, number of kernel rows per cob and test weight. The above result clearly indicates the importance of site specific application of nutrients required to achieve the targeted yields of maize. The increase in grain yield of maize was due to the application of higher level inorganic fertilizers. These results are in accordance with those obtained by Jayaprakash et al., (2006); Jemal Abdulai (2010); Biradar et al., (2013) and Pagad (2014).The interaction data on grain yield revealed that, $900 \mathrm{M}$ GOLD with target yield of $140 \mathrm{q} \mathrm{ha}^{-1}\left(130.53 \mathrm{q} \mathrm{ha}^{-1}\right)$ recorded significantly higher grain yield over rest of the treatments (Table 1). However, the lower grain yield was recorded in NK-6240 with absolute control (27.56 q ha $\left.{ }^{-1}\right)$.

\section{Growth parameters}

The data on plant height of maize shows significant difference between the two hybrids at different phonological stages except 25DAS (Table 2). It is evident, from the table that, the maize hybrid NK-6240 recorded significantly, higher plant height (32.48, $173.52,176.24,187.67,193.56 \mathrm{~cm}$ at 35,55 , 65,85 DAS and harvest, respectively) over 900 M GOLD $(31.85,168.52$, 171.82, 184.22, $190.52 \mathrm{~cm}$ at $35,55,65,85$ DAS and harvest, respectively).This might be due to genetic makeup and morphological characteristics of hybrids in exploiting climatic optima at important growth stages (Jemal Abdulai, 2010).Among different target yield levels significantly higher plant height was recorded with targeted yield level of $140 \mathrm{q} \mathrm{ha}^{-1}$ (14.62, 
$22.51,123.41,126.40,133.90,140.44 \mathrm{~cm}$ at $25,35,55,65,85$ DAS and harvest, respectively) over target yield of $100,120 \mathrm{q}$ $\mathrm{ha}^{-1}$, recommended dose of fertilizer (RDF) and absolute control. Significantly, a shorter plant height (13.14, 20.09, 103.37, 104.67, 107.17, 107.76cmat 35, 55, 65, 85 DAS and harvest, respectively) was recorded in absolute control. This improved vegetative growth has laid down foundation for better infrastructure for dry matter production and ultimately lead to better yield of the crop. The higher nutrient uptake right from in early stage of crop growth may be one of the reasons for improved vegetative growth. The interaction data on plant height revealed that, the maize hybrid NK-6240 with targeted yield of $140 \mathrm{q} \mathrm{ha}^{-1}(22.40,34.32,187.03,191.03$, $203.32,212.47 \mathrm{~cm}$ at $25,35,55,65,85$ DAS and harvest, respectively) recorded significantly, higher plant height over rest of the treatments. However, the lower plant height was recorded in $900 \mathrm{M}$ GOLD with absolute control (19.47, 29.97, 151.67, $154.17,158.92,160.13 \mathrm{~cm}$ at $25,35,55,65$, 85 DAS and harvest, respectively).The higher total plant height might be due to higher dry matter accumulation in stem, leaves and reproductive parts at all the growth stages and it is the reflection of photosynthetic efficiency of cultivar at the nutrition level supplied.

Both the hybrids failed to show any significant differences, with respect to number of leaves at different phonological stages (Table 2). Significantly higher number of leaves was recorded in targeted yield level of $140 \mathrm{q} \mathrm{ha}^{-1}(3.33,4.96,7.67,9.24,7.24$, 2.36 per plant at $25,35,55,65,85$ DAS and harvest, respectively) over target yield of 100 , $120 \mathrm{q} \mathrm{ha}^{-1}$, recommended dose of fertilizer (RDF) and absolute control among different target yield levels. Significantly lower number of leaves $(2.62,3.73,6.62,8.09,6.09$, 1.20 per plant at $25,35,55,65,85 \mathrm{DAS}$ and harvest, respectively) was observed in absolute control. The higher nutrient uptake right from in early stage of crop growth may be one of the reasons for improved vegetative growth. Interaction show significant difference between maize hybrids and targeted yields at different phonological stages except 25 DAS. The data on interaction effect on number of leaves revealed that, 900 M GOLD with targeted yield of $140 \mathrm{q} \mathrm{ha}^{-1}$ (7.93, 11.93, 14.20, 11.20, 3.87 per plant at $35,55,65,85$ DAS and harvest, respectively) recorded significantly, higher number of leaves over rest of the treatments.

However, the lower number of leaves was recorded in NK-6240 with absolute control $(5.00,9.47,11.80,8.80,1.47$ per plant at 35, $55,65,85$ DAS and harvest, respectively).The higher number of leaves might be due to higher dry matter leaves and reproductive parts at all the growth stages and it is the reflection of photosynthetic efficiency of cultivar at the nutrition level supplied.

Results showed that the leaf area (Table 3) tended to increase up to 65 DAS beyond which it declined towards harvest, which was due to senescence of foliage. The data on leaf area of maize shows significant difference between the two hybrids at different phonological stages except 25DAS.Significantly higher leaf area per plant was recorded in $900 \mathrm{M}$ GOLD (1662.99, $4610.06,6057.73,2909.20 \mathrm{~cm}^{2}$ at $35,55,65$, 85 DAS, respectively) over NK-6240 (1279.0, $3769.75,5068027,2268.66 \mathrm{~cm}^{2}$ at $35,55,65$, 85 DAS, respectively). These results corroborate with the findings of Jemal Abdulai (2010). Plant canopies intercept light with varying degrees of efficiency associated chiefly with the leaf area. The efficiency of intercepting of incident light, combined with efficiency of photochemical reactions of the leaves determine the efficiency of the canopy in utilizing radiation energy per unit of land area. 
Table.1 Grain yield ( $\mathrm{q} \mathrm{ha}^{-1}$ ) of maize hybrids as influenced by target yield approach

$\mathrm{T}_{1}$ :

\begin{tabular}{|c|c|}
\hline Treatments & Grain yield (q ha $\left.{ }^{-1}\right)$ \\
\hline \multicolumn{2}{|l|}{ Hybrids (H) } \\
\hline $\mathrm{H}_{1}-\mathrm{NK} 6240$ & 91.81 \\
\hline $\mathrm{H}_{2}-900 \mathrm{M}$ GOLD & 93.47 \\
\hline S.Em \pm & 0.11 \\
\hline $\mathrm{CD}(0.05)$ & 0.64 \\
\hline \multicolumn{2}{|l|}{ Target yields $\left(\mathrm{q} \mathrm{ha}^{-1}\right)(\mathrm{T})$} \\
\hline $\mathrm{T}_{1}$ - Target yield $100 \mathrm{q} \mathrm{ha}^{-1}$ & 71.75 \\
\hline $\mathrm{T}_{2}$ - Target yield $120 \mathrm{q} \mathrm{ha}^{-1}$ & 79.70 \\
\hline $\mathrm{T}_{3}$ - Target yield $140 \mathrm{q} \mathrm{ha}^{-1}$ & 86.34 \\
\hline $\mathrm{T}_{4}-\mathrm{RDF}$ & 52.00 \\
\hline $\mathrm{T}_{5}$ - Absolute control & 19.01 \\
\hline S.Em \pm & 0.24 \\
\hline $\mathrm{CD}(0.05)$ & 0.72 \\
\hline \multicolumn{2}{|l|}{ Interactions $(\mathbf{H} \times \mathbf{T})$} \\
\hline $\mathrm{H}_{1} \mathrm{~T}_{1}$ & 106.35 \\
\hline $\mathrm{H}_{1} \mathrm{~T}_{2}$ & 119.27 \\
\hline $\mathrm{H}_{1} \mathrm{~T}_{3}$ & 128.49 \\
\hline $\mathrm{H}_{1} \mathrm{~T}_{4}$ & 77.39 \\
\hline $\mathrm{H}_{1} \mathrm{~T}_{5}$ & 27.56 \\
\hline $\mathrm{H}_{2} \mathrm{~T}_{1}$ & 108.90 \\
\hline $\mathrm{H}_{2} \mathrm{~T}_{2}$ & 119.82 \\
\hline $\mathrm{H}_{2} \mathrm{~T}_{3}$ & 130.53 \\
\hline $\mathrm{H}_{2} \mathrm{~T}_{4}$ & 78.62 \\
\hline $\mathrm{H}_{2} \mathrm{~T}_{5}$ & 29.46 \\
\hline $\begin{array}{l}\text { S.Em } \pm \\
\text { CD }(0.05)\end{array}$ & $\begin{array}{l}0.34 \\
0.72 \\
\end{array}$ \\
\hline
\end{tabular}

288.0:89.0:67.2 N, $\mathrm{P}_{2} \mathrm{O}_{5}$ and $\mathrm{K}_{2} \mathrm{O} \mathrm{kg} \mathrm{ha}{ }^{-1} \quad ; \mathrm{T}_{2}$ : 345.6:106.8:80.6 N, $\mathrm{P}_{2} \mathrm{O}_{5}$ and $\mathrm{K}_{2} \mathrm{O} \mathrm{kg} \mathrm{ha}{ }^{-1}$ $\mathrm{T}_{3}$ : 403.2:124.6:94.0 N, $\mathrm{P}_{2} \mathrm{O}_{5}$ and $\mathrm{K}_{2} \mathrm{O} \mathrm{kg} \mathrm{ha}{ }^{-1} ; \mathrm{T}_{4}: 150.0: 65.0: 65.0 \mathrm{~N}, \mathrm{P}_{2} \mathrm{O}_{5}$ and $\mathrm{K}_{2} \mathrm{O} \mathrm{kg} \mathrm{ha}^{-1}$ 
Table.2 Plant height and number of green leaves per plant of maize hybrids as influenced by target yield approach

\begin{tabular}{|c|c|c|c|c|c|c|c|c|c|c|c|c|}
\hline \multirow[b]{2}{*}{ Treatments } & \multicolumn{6}{|c|}{ Plant height $(\mathrm{cm})$} & \multicolumn{6}{|c|}{ Number of green leaves plant ${ }^{-1}$} \\
\hline & $\begin{array}{c}25 \\
\text { DAS }\end{array}$ & $\begin{array}{c}35 \\
\text { DAS } \\
\end{array}$ & 55 DAS & 65 DAS & 85 DAS & $\begin{array}{c}\text { At } \\
\text { harvest }\end{array}$ & $\begin{array}{c}25 \\
\text { DAS }\end{array}$ & 35 DAS & 55 DAS & 65 DAS & 85 DAS & $\begin{array}{c}\text { At } \\
\text { harvest }\end{array}$ \\
\hline \multicolumn{13}{|l|}{ Hybrids (H) } \\
\hline $\mathrm{H}_{1}-\mathrm{NK} 6240$ & 21.07 & 32.48 & 173.52 & 176.24 & 187.67 & 193.56 & 4.32 & 5.92 & 10.17 & 12.52 & 9.52 & 2.19 \\
\hline $\mathrm{H}_{2}-900 \mathrm{M}$ GOLD & 20.33 & 31.85 & 168.52 & 171.82 & 184.22 & 190.52 & 4.48 & 6.96 & 10.99 & 13.19 & 10.19 & 2.85 \\
\hline S.Em \pm & 0.15 & 0.09 & 0.32 & 0.31 & 0.02 & 0.20 & 0.57 & 0.03 & 0.24 & 0.53 & 0.53 & 0.53 \\
\hline $\mathrm{CD}(0.05)$ & NS & 0.55 & 1.93 & 1.89 & 0.14 & 1.20 & NS & NS & NS & NS & NS & NS \\
\hline \multicolumn{13}{|l|}{ Target yields $\left(q\right.$ ha $\left.^{-1}\right)(\mathrm{T})$} \\
\hline $\mathrm{T}_{1}$ - Target yield $100 \mathrm{q} \mathrm{ha}^{-1}$ & 13.63 & 21.48 & 114.13 & 116.13 & 125.71 & 130.08 & 2.89 & 4.33 & 6.93 & 8.44 & 6.44 & 1.56 \\
\hline $\mathrm{T}_{2}$ - Target yield $120 \mathrm{q} \mathrm{ha}^{-1}$ & 14.17 & 22.01 & 117.47 & 119.48 & 129.61 & 134.44 & 3.07 & 4.51 & 7.20 & 8.76 & 6.76 & 1.87 \\
\hline $\mathrm{T}_{3}$ - Target yield $140 \mathrm{q} \mathrm{ha}^{-1}$ & 14.62 & 22.51 & 123.41 & 126.40 & 133.98 & 140.44 & 3.33 & 4.96 & 7.67 & 9.24 & 7.24 & 2.36 \\
\hline $\mathrm{T}_{4}-\mathrm{RDF}$ & 13.42 & 21.12 & 111.68 & 113.42 & 123.34 & 127.21 & 2.76 & 3.93 & 6.84 & 8.31 & 6.31 & 1.42 \\
\hline $\mathrm{T}_{5}$ - Absolute control & 13.14 & 20.09 & 103.37 & 104.67 & 107.17 & 107.96 & 2.62 & 3.73 & 6.62 & 8.09 & 6.09 & 1.20 \\
\hline S.Em \pm & 0.20 & 0.07 & 0.51 & 0.50 & 0.29 & 0.43 & 0.02 & 0.08 & 0.06 & 0.05 & 0.05 & 0.05 \\
\hline $\mathrm{CD}(0.05)$ & 0.61 & 0.21 & 1.53 & 1.51 & 0.87 & 1.28 & 0.05 & 0.23 & 0.17 & 0.15 & 0.15 & 0.15 \\
\hline \multicolumn{13}{|l|}{ Interactions $(\mathrm{H} \times \mathbf{T})$} \\
\hline $\mathrm{H}_{1} \mathrm{~T}_{1}$ & 20.73 & 32.42 & 172.20 & 175.80 & 190.02 & 196.77 & 4.27 & 6.00 & 10.00 & 12.33 & 9.33 & 2.00 \\
\hline $\mathrm{H}_{1} \mathrm{~T}_{2}$ & 21.77 & 33.42 & 179.98 & 182.13 & 196.32 & 203.27 & 4.60 & 6.27 & 10.40 & 12.80 & 9.80 & 2.47 \\
\hline $\mathrm{H}_{1} \mathrm{~T}_{3}$ & 22.40 & 34.32 & 187.03 & 191.30 & 203.32 & 212.47 & 4.80 & 6.93 & 11.07 & 13.53 & 10.53 & 3.20 \\
\hline $\mathrm{H}_{1} \mathrm{~T}_{4}$ & 20.47 & 31.95 & 169.90 & 172.13 & 186.12 & 191.57 & 4.07 & 5.40 & 9.93 & 12.13 & 9.13 & 1.80 \\
\hline $\mathrm{H}_{1} \mathrm{~T}_{5}$ & 19.97 & 30.32 & 158.47 & 159.83 & 162.58 & 163.75 & 3.87 & 5.00 & 9.47 & 11.80 & 8.80 & 1.47 \\
\hline $\mathrm{H}_{2} \mathrm{~T}_{1}$ & 20.17 & 32.03 & 170.20 & 172.60 & 187.12 & 193.47 & 4.40 & 7.00 & 10.80 & 13.00 & 10.00 & 2.67 \\
\hline $\mathrm{H}_{2} \mathrm{~T}_{2}$ & 20.73 & 32.61 & 172.43 & 176.30 & 192.52 & 200.07 & 4.60 & 7.27 & 11.20 & 13.47 & 10.47 & 3.13 \\
\hline $\mathrm{H}_{2} \mathrm{~T}_{3}$ & 21.47 & 33.22 & 183.20 & 187.90 & 198.62 & 208.87 & 5.20 & 7.93 & 11.93 & 14.20 & 11.20 & 3.87 \\
\hline $\mathrm{H}_{2} \mathrm{~T}_{4}$ & 19.80 & 31.41 & 165.13 & 168.13 & 183.92 & 190.07 & 4.20 & 6.40 & 10.60 & 12.80 & 9.80 & 2.47 \\
\hline $\mathrm{H}_{2} \mathrm{~T}_{5}$ & 19.47 & 29.97 & 151.65 & 154.17 & 158.92 & 160.13 & 4.00 & 6.20 & 10.40 & 12.47 & 9.47 & 2.13 \\
\hline S.Em \pm & 0.29 & 0.10 & 0.72 & 0.71 & 0.41 & 0.60 & 0.03 & 0.11 & 0.08 & 0.07 & 0.07 & 0.07 \\
\hline $\mathrm{CD}(0.05)$ & NS & 0.21 & 1.53 & 1.51 & 0.87 & 1.28 & NS & 0.23 & 0.17 & 0.15 & 0.15 & 0.15 \\
\hline
\end{tabular}


Table.3 Leaf area of maize hybrids as influenced by target yield approach

\begin{tabular}{|c|c|c|c|c|c|c|c|c|c|}
\hline \multirow{2}{*}{ Treatments } & \multicolumn{5}{|c|}{ Leaf area $\left(\mathrm{cm}^{2}\right)$} & \multicolumn{4}{|c|}{ Leaf area duration (LAD) } \\
\hline & 25 DAS & 35 DAS & 55 DAS & 65 DAS & 85 DAS & 25-35 DAS & 35-55 DAS & 55-65 DAS & 65-85 DAS \\
\hline \multicolumn{10}{|l|}{ Hybrids (H) } \\
\hline $\mathrm{H}_{1}-\mathrm{NK} 6240$ & 697.39 & 1279.00 & 3769.75 & 5068.27 & 2268.66 & 8.23 & 42.07 & 36.83 & 61.14 \\
\hline $\mathrm{H}_{2}-900 \mathrm{M}$ GOLD & 753.52 & 1662.99 & 4610.06 & 6057.73 & 2909.20 & 10.07 & 52.28 & 44.45 & 74.72 \\
\hline S.Em \pm & 97.51 & 2.18 & 79.76 & 213.40 & 121.00 & 0.41 & 0.65 & 1.22 & 2.79 \\
\hline $\mathrm{CD}(0.05)$ & NS & 13.24 & 485.33 & 1298.53 & 736.30 & NS & 3.98 & 7.43 & 16.96 \\
\hline \multicolumn{10}{|l|}{ Target yields $\left(\mathrm{q} \mathrm{ha}^{-1}\right)(\mathrm{T})$} \\
\hline $\mathrm{T}_{1}$ - Target yield $100 \mathrm{q}^{-} \mathrm{ha}^{-}$ & 470.14 & 990.08 & 2736.26 & 3679.22 & 1605.24 & 6.08 & 31.05 & 26.73 & 44.04 \\
\hline $\mathrm{T}_{2}$ - Target yield $120 \mathrm{q} \mathrm{ha}^{-}$ & 520.44 & 1064.03 & 2919.48 & 3912.77 & 1885.41 & 6.60 & 33.20 & 28.47 & 48.32 \\
\hline $\mathrm{T}_{3}$ - Target yield $140 \mathrm{q} \mathrm{ha}^{-}$ & 610.04 & 1255.40 & 3402.63 & 4486.95 & 2406.92 & 7.77 & 38.82 & 32.87 & 57.45 \\
\hline $\mathrm{T}_{4}-\mathrm{RDF}$ & 436.48 & 831.95 & 2640.34 & 3542.10 & 1526.89 & 5.29 & 28.94 & 25.76 & 42.24 \\
\hline $\mathrm{T}_{5}$ - Absolute control & 381.09 & 761.85 & 2267.66 & 2922.29 & 1205.30 & 4.76 & 25.25 & 21.62 & 34.40 \\
\hline S.Em \pm & 12.16 & 26.93 & 41.88 & 71.47 & 47.00 & 0.13 & 0.50 & 0.40 & 0.98 \\
\hline $\mathrm{CD}(0.05)$ & 36.45 & 80.75 & 125.56 & 214.28 & 140.91 & 0.39 & 1.49 & 1.19 & 2.92 \\
\hline \multicolumn{10}{|l|}{ Interactions $(\mathrm{H} \times \mathrm{T})$} \\
\hline $\mathrm{H}_{1} \mathrm{~T}_{1}$ & 686.83 & 1284.31 & 3722.84 & 5030.19 & 2091.28 & 8.21 & 41.73 & 36.47 & 59.35 \\
\hline $\mathrm{H}_{1} \mathrm{~T}_{2}$ & 759.00 & 1386.23 & 3940.47 & 5318.76 & 2465.19 & 8.94 & 44.39 & 38.58 & 64.87 \\
\hline $\mathrm{H}_{1} \mathrm{~T}_{3}$ & 842.52 & 1598.34 & 4569.89 & 6152.72 & 3220.72 & 10.17 & 51.40 & 44.68 & 78.11 \\
\hline $\mathrm{H}_{1} \mathrm{~T}_{4}$ & 640.91 & 1110.01 & 3604.79 & 4841.27 & 1987.30 & 7.30 & 39.29 & 35.19 & 56.90 \\
\hline $\mathrm{H}_{1} \mathrm{~T}_{5}$ & 557.71 & 1016.13 & 3010.78 & 3998.40 & 1578.79 & 6.56 & 33.56 & 29.20 & 46.48 \\
\hline $\mathrm{H}_{2} \mathrm{~T}_{1}$ & 723.58 & 1685.93 & 4485.93 & 6007.47 & 2724.43 & 10.04 & 51.43 & 43.72 & 72.77 \\
\hline $\mathrm{H}_{2} \mathrm{~T}_{2}$ & 802.32 & 1805.87 & 4817.96 & 6419.56 & 3191.04 & 10.87 & 55.20 & 46.82 & 80.09 \\
\hline $\mathrm{H}_{2} \mathrm{~T}_{3}$ & 987.61 & 2167.86 & 5638.00 & 7308.14 & 4000.04 & 13.15 & 65.05 & 53.94 & 94.23 \\
\hline $\mathrm{H}_{2} \mathrm{~T}_{4}$ & 668.54 & 1385.83 & 4316.22 & 5785.03 & 2593.36 & 8.56 & 47.52 & 42.09 & 69.82 \\
\hline $\mathrm{H}_{2} \mathrm{~T}_{5}$ & 585.55 & 1269.43 & 3792.21 & 4768.48 & 2037.11 & 7.73 & 42.18 & 35.67 & 56.71 \\
\hline S.Em \pm & 17.19 & 38.09 & 59.23 & 101.08 & 66.47 & 0.18 & 0.70 & 0.56 & 1.38 \\
\hline $\mathrm{CD}(0.05)$ & 36.45 & 80.75 & 125.56 & 214.28 & 140.91 & 0.39 & 1.49 & 1.19 & 2.92 \\
\hline
\end{tabular}


Table.4 Stem girth of maize hybrids as influenced by target yield approach

\begin{tabular}{|c|c|c|c|c|c|}
\hline \multirow{2}{*}{ Treatments } & \multicolumn{5}{|c|}{ Stem girth $(\mathrm{cm})$} \\
\hline & 25 DAS & 35 DAS & 55 DAS & 65 DAS & 85 DAS \\
\hline \multicolumn{6}{|l|}{ Hybrids (H) } \\
\hline $\mathrm{H}_{1}-\mathrm{NK} 6240$ & 3.01 & 4.02 & 7.42 & 7.59 & 7.90 \\
\hline $\mathrm{H}_{2}-900 \mathrm{M}$ GOLD & 3.03 & 4.16 & 7.74 & 7.63 & 7.94 \\
\hline S.Em & 0.02 & 0.01 & 0.05 & 0.16 & 0.18 \\
\hline $\mathrm{CD}(0.05)$ & $\mathrm{NS}$ & 0.09 & 0.29 & 0.96 & 1.04 \\
\hline \multicolumn{6}{|l|}{ Target yields $\left(q h^{-1}\right)(T)$} \\
\hline $\mathrm{T}_{1}$ - Target yield $100 \mathrm{q} \mathrm{ha}^{-1}$ & 2.00 & 2.75 & 5.07 & 5.02 & 5.26 \\
\hline $\mathrm{T}_{2}$ - Target yield $120 \mathrm{q} \mathrm{ha}^{-1}$ & 2.07 & 2.83 & 5.30 & 5.35 & 5.58 \\
\hline $\mathrm{T}_{3}$ - Target yield $140 \mathrm{q} \mathrm{ha}^{-1}$ & 2.15 & 2.99 & 5.74 & 5.78 & 6.02 \\
\hline $\mathrm{T}_{4}-\mathrm{RDF}$ & 1.97 & 2.59 & 4.84 & 4.85 & 5.09 \\
\hline $\mathrm{T}_{5}-$ Absolute control & 1.88 & 2.46 & 4.30 & 4.36 & 4.44 \\
\hline S.Em \pm & 0.01 & 0.02 & 0.03 & 0.07 & 0.09 \\
\hline $\mathrm{CD}(0.05)$ & 0.04 & 0.07 & 0.10 & 0.21 & 0.27 \\
\hline \multicolumn{6}{|l|}{ Interactions $(\mathbf{H} \times \mathbf{T})$} \\
\hline $\mathrm{H}_{1} \mathrm{~T}_{1}$ & 2.99 & 4.04 & 7.26 & 7.51 & 7.86 \\
\hline $\mathrm{H}_{1} \mathrm{~T}_{2}$ & 3.08 & 4.20 & 7.84 & 8.06 & 8.41 \\
\hline $\mathrm{H}_{1} \mathrm{~T}_{3}$ & 3.21 & 4.47 & 8.54 & 8.60 & 8.95 \\
\hline $\mathrm{H}_{1} \mathrm{~T}_{4}$ & 2.95 & 3.72 & 7.04 & 7.25 & 7.60 \\
\hline $\mathrm{H}_{1} \mathrm{~T}_{5}$ & 2.81 & 3.65 & 6.40 & 6.53 & 6.66 \\
\hline $\mathrm{H}_{2} \mathrm{~T}_{1}$ & 3.02 & 4.20 & 7.96 & 7.57 & 7.92 \\
\hline $\mathrm{H}_{2} \mathrm{~T}_{2}$ & 3.12 & 4.29 & 8.05 & 7.99 & 8.34 \\
\hline $\mathrm{H}_{2} \mathrm{~T}_{3}$ & 3.25 & 4.51 & 8.68 & 8.75 & 9.10 \\
\hline $\mathrm{H}_{2} \mathrm{~T}_{4}$ & 2.96 & 4.07 & 7.48 & 7.31 & 7.66 \\
\hline $\mathrm{H}_{2} \mathrm{~T}_{5}$ & 2.82 & 3.72 & 6.51 & 6.54 & 6.67 \\
\hline S.Em \pm & 0.02 & 0.03 & 0.05 & 0.10 & 0.11 \\
\hline $\mathrm{CD}(0.05)$ & $\mathrm{NS}$ & 0.07 & 0.10 & 0.28 & 0.29 \\
\hline
\end{tabular}


Similarly, leaf area was influenced markedly due to target yield based fertilizer application. Increasing target yield levels from 100 to $140 \mathrm{q}$ $\mathrm{ha}^{-1}$ significantly increased leaf area at all the stages. Among different target yield levels, significantly, higher leaf area per plant was recorded with targeted yield level of $140 \mathrm{q} \mathrm{ha}^{-1}$ (610.04, 1255.40, 3402.63, 4486.95, 2406.92 $\mathrm{cm}^{2}$ at $25,35,55,65,85$ DAS, respectively) over target yield of $100,120 \mathrm{q} \mathrm{ha}^{-1}$, recommended dose of fertilizer (RDF) and absolute control. Significantly, a lower leaf area per plant (381.09, 761.85, 2267.66, 2922.29, 1205.30 $\mathrm{cm}^{2}$ at 25, 35, 55, 65, 85 DAS, respectively) was observed in absolute control. At different growth periods, application of higher doses of nitrogen favored the crop to put forth more leaf area. The interaction data on leaf area per plant revealed that, $900 \mathrm{M}$ GOLD with target yield of $140 \mathrm{q} \mathrm{ha} \mathrm{ha}^{-1}(987.61,2167.8$, 5638, 7308.14, $4000.04 \mathrm{~cm}^{2}$ at 25, 35, 55, 65 and 85 DAS, respectively) recorded significantly, higher leaf area per plant over rest of the treatments. However, the lower leaf area per plant was recorded in NK-6240 with absolute control (557.71, 1016.13, 3010.78, 3998.40, 1578.79 $\mathrm{cm}^{2}$ at 25, 35, 55, 65 and 85 DAS, respectively).The present findings are in conformity with those of Jemal Abdulai (2010).

The data on leaf area duration of maize shows significant difference between the two hybrids at different phonological stages except between 25- 35DAS (Table 3). Significantly higher leaf duration was recorded in $900 \mathrm{M}$ GOLD (52.28, 44.45, 74.72 between 35-55, 55-65 and 65-85, respectively) over NK-6240 (42.07, 36.83, 61.14 between 35-55, 55-65 and 65-85, respectively). These results corroborate the findings of Jemal Abdulai (2010) and Murthy (2014). Plant canopies intercept light with varying degrees of efficiency associated chiefly with the LAI. The efficiency of intercepting of incident light, combined with efficiency of photochemical reactions of the leaves determine the efficiency of the canopy in utilizing radiation energy per unit of land area. Among different target yield levels, significantly, higher LAD was recorded with targeted yield level of
$140 \mathrm{q} \mathrm{ha}^{-1}(7.77,38.82,32.87,57.45$ between $25-35,35-55,55-65$ and 65-85, respectively) over target yield of $100,120 \mathrm{q} \mathrm{ha}$, recommended dose of fertilizer (RDF) and absolute control. Significantly lower LAD (4.76, 25.25, 21.62, 34.40between 35-55, 55-65 and $65-85$, respectively) was observed in absolute control. The relevance of higher LAD was clearly brought out by Watson (1952). According to whom the formation of optimum photosynthetic area and maintaining the leaf photo synthetically active stage for longer period were essential for increasing grain yield. The improved photosynthetic capacity was associated with higher $\mathrm{N}, \mathrm{P}_{2} \mathrm{O}_{5}$ and $\mathrm{K}_{2} \mathrm{O}$ nutrition as indicated by better uptake of major nutrients. All the three elements $\left(\mathrm{N}, \mathrm{P}_{2} \mathrm{O}_{5}\right.$ and $\mathrm{K}_{2} \mathrm{O}$ ) are critically involved in photosynthesis and dry matter production (Tsidale et al., 1986). The interaction data on LAD revealed that, $900 \mathrm{M}$ GOLD with targeted yield of $140 \mathrm{q}$ ha $^{-1}(13.15,65.05,53.94,94.23$ between 25 to 35 DAS, 35 to 55 DAS, 55 to 65 DAS and 65 to 85 DAS, respectively) recorded significantly higher leaf area duration over rest of the treatments. However, the LAD was recorded in NK-6240 with absolute control $(6.56,33.56$, $29.20,46.48$ between 25 to 35 DAS, 35 to 55 DAS, 55 to 65 DAS and 65 to 85 DAS, respectively).The present findings are in conformity with those of Jemal Abdulai (2010).

Stem girth are important growth parameters which influence carbon storage and its subsequent utilization for grain filling in maize. These are cases where the utilization of stem reserves for grain filling in constitutive irrespective of environmental conditions (Borrel et al., 1993). The data on stem girth of maize shows significant difference between the two hybrids at different phonological stages except 25DAS (Table 4). Significantly higher stem girth was recorded in $900 \mathrm{M}$ GOLD $(4.16,7.74$, $7.63,7.94 \mathrm{~cm}$ at $35,55,65,85 \mathrm{DAS}$, respectively) over NK-6240 (4.02, 7.42, 7.59, $7.90 \mathrm{~cm}$ at35, 55, 65, 85 DAS, respectively).Similar results were also have been revealed by Jemal Abdulai (2010) and Daikho (2013). Among different target yield 
levels, significantly, higher stem girth was recorded with targeted yield level of $140 \mathrm{q} \mathrm{ha}^{-1}$ $(2.15,2.99,5.74,5.78,6.02 \mathrm{~cm}$ at $25,35,55$, 65,85 DAS, respectively) over target yield of $100,120 \mathrm{q} \mathrm{ha}^{-1}$, recommended dose of fertilizer (RDF) and absolute control. Significantly, lower stem girth $(1.88,2.46,4.30,4.36,4.40 \mathrm{~cm}$ at $35,55,65,85$ DAS, respectively) was observed in absolute control.

This improved vegetative growth has laid down foundation for better infrastructure for dry matter production and ultimately lead to better yield of the crop. The higher nutrient uptake right from in early stage of crop growth may be one of the reasons for improved vegetative growth. The data on the interaction between maize hybrids and target yields show significant difference at different phonological stages except 25 DAS.

The interaction data on stem girth revealed that, $900 \mathrm{M}$ GOLD with target yield of $140 \mathrm{q} \mathrm{ha}^{-1}$ $(4.51,8.68,8.75,9.10 \mathrm{~cm}$ at $35,55,65,85$ DAS, respectively) recorded significantly, higher stem girth over rest of the treatments. However, the lower stem girth was recorded in NK-6240 with absolute control (3.65, 6.40, $6.53,6.66 \mathrm{~cm}$ at $35,55,65,85 \mathrm{DAS}$, respectively).Higher stem girth was a cumulative effect of total dry matter production and its translocation and accumulation in stem. These results are agreement with Jemal Abdulai (2010).

\section{References}

Biradar, A., Jayadeva, H. M., Shankarlingappa, B. C and Vishwanath, A. P. 2013. Effect of target yield approach on growth, yield and nutrient uptake at flowering of maize. Mysore J. Agric. Sci., 47(4): 707-712.

Borrel, A. K., Incoll, L. D. and Dalling, 1993. The influence of the rhtl and rht2 alleles on the deposition and use of stem reserves in wheat. Ann. Bot., 71: 317-326.

Daikho, A., 2013. Performance of maize hybrids to varying fertilizer levels in the northern transitional zone of Karnataka. M. Sc. (Agri.) Thesis, Univ. Agric. Sci., Dharwad, Karnataka (India).

Hirpa, D. D., Hunshal, C. S., Hiremath, S. M., Awaknavar, J. S., Wali, M. C., Nadagouda, B. T and Chandrashekar, C. P. 2013. Performance of maize (Zea mays L.) hybrids as influenced by different levels of nitrogen, phosphorus, potassium and sulphur application. Karnataka J. Agric. Sci. 26(2), 194-199.

Jayaprakash, T. C., Nagalikar, V. P., Pujari, B. T. and Shetty, R. A. 2006. Effect of organics and inorganics on growth and yield of maize under irrigation. Karnataka J. Agric. Sci., 18(3): 798.

Jemal Abdulai, 2010. Response of maize (Zea mays L.) and chickpea (Cicerarietinum L.) to site specific nutrient management (SSNM) through targeted yield approach, M. Sc. (Agri.) Thesis, Univ. Agric. Sci., Dharwad, Karnataka (India).

Murthy, B., 2014. Evaluation of maize genotypes for moisture stress condition. M. Sc. (Agri.) Thesis, Univ. Agric. Sci., Dharwad, Karnataka (India).

Pagad, S., 2014. Precision nutrient management in maize. M. Sc. (Agri.) Thesis, Univ. Agric. Sci., Dharwad, Karnataka (India).

Singh, P., Shukla, U. N., Kaushal K, Smita S, Kumar, V and Rajeev, K. 2014. Evaluation of growth, yield and quality of maize as influenced by genotypes and nitrogen levels. Bangladesh J. Bot.43 (1), 59-64.

Tisdale, S. L., Nelson, W and Beaton, J., 1986. Soil fertility and fertilizers. The Mac Millan Publication Campany, New York, pp: 634641.

Watson, D. J., 1952. The physiological basis for variation in yield. Advances in Agron, 4: 101-145.

\section{How to cite this article:}

Chetan, H.T., M.P. Potdar, B.T. Nadagouda and Gurupada B. Balol. 2017. Yield and Growth Parameters of Maize Hybrids As Influenced By Target Yield Approach. Int.J.Curr.Microbiol.App.Sci. 6(9): 220-229. doi: https://doi.org/10.20546/ijcmas.2017.609.030 